\title{
Estimating short time interval densities in a CTM-KF model
}

\author{
Arlinda Alimehaj Rrecaj ${ }^{*}$, , Marija Malenkovska Todorova ${ }^{2}$ \\ ${ }^{1}$ University of Prishtina, Mechanical Engineering Faculty, Traffic and Transportation Department, 10000 Prishtina, Kosovo \\ ${ }^{2}$ University of St.Klement Ohrid, Faculty of Technical Sciences, Transport Engineering, 7000 Bitola, Republic of Macedonia
}

\author{
A R T I C L E I N F O \\ Article history: \\ Received: 27 January, 2018 \\ Accepted: 21 February, 2018 \\ Online: 08 March, 2018
}

Keywords:

Cell

Density

Kalman Filter

\begin{abstract}
A B S T R A C T
On-ramping is being widely used as e method to increase the freeway operational efficiency. The main traffic parameter that must be taken in consideration for the implementation of the feedback control strategies for the on-ramp metering is density on the main section of road. In this paper is given discretized model of traffic which is then improved by a recursive technique called Kalman-Filter with the aid of which is possible to predict the density, by only having the traffic flow measured on the start and end road section. Kalman Filter is based on linear relationship of flow and density. By minimizing the square of error between of the measurements and the estimated values of flows, a gain is derived which then is applied to the densities of the model in order to obtain the greatest accuracy of these values.
\end{abstract}

\section{Introduction}

The increasing demand of motorized vehicles is becoming one of the major problems that face the developed world places in nowadays. Based on some statistics in Great Britain, $90 \%$ of the journeys are by road, during the last decade, and for more the road distances travelled, have increased by over $1000 \%$ on the last sixty years. [1]

On-ramp metering [2] is being widely used as e method to increase the freeway operational efficiency by regulating the traffic interruption by minor roads, while maintaining the right of way on the major section until it reaches me critical densities, which assure the maximal flow [3]. Measure of the density on the major road is difficult to measure. In this paper is proposed a discretized traffic model (CTM) to obtain traffic densities which then will be accurate through Kalman Filter [4, 5 and 6].

\section{CTM model of a Highway with three cells}

If we denote with $\rho_{i}(\mathrm{k})$ the density of a cell (uniform or nonuniform length), instead of the number of vehicles $n_{i}$ on the a unit length cell, then we can bring equation (1.1)[6].

for density $\rho_{i}(k+1)$ of the cell $i$ updated time step in $(k+1)$, where $T_{s}$ is the discrete time unit in seconds.

\footnotetext{
*Arlinda Alimehaj Rrecaj, Email: arlinda.alimehaj@uni-pr.edu
}

$$
\rho_{i}(k+1)=\rho_{i}(k)+\frac{T_{s}}{L}\left(q_{i}(k)-q_{i+1}(k)\right)
$$

Analyzing a highway partitioned in three cells (for the sake of simply illustration) with an on ramp and an off ramp, by assuming that the belonging cells can be in the free flow either in congested mode the densities on each cell can be written as in equations (1.2, $1.3,1.4)$.

The densities on each cell are:

$$
\begin{gathered}
\rho_{i-1}(k+1)=\rho_{i-1}(k)+\frac{T_{s}}{L}\left(q_{i-1}(k)-q_{i}(k)+r(k)\right) \text { or } \\
\rho_{1}(k+1)=\rho_{1}(k)+\frac{T_{s}}{L}\left(q_{1}(k)-q_{2}(k)+r(k)\right) \\
\rho_{i}(k+1)=\rho_{i}(k)+\frac{T_{s}}{L}\left(q_{i}(k)-q_{i+1}(k)\right) \text { or } \\
\rho_{2}(k+1)=\rho_{2}(k)+\frac{T_{s}}{L}\left(q_{2}(k)-q_{3}(k)\right) \\
\rho_{i+1}(k+1)=\rho_{i+1}(k)+\frac{T_{s}}{L}\left(q_{i+1}(k)-q_{4}(k)-f(k)\right) \text { or } \\
\rho_{3}(k+1)=\rho_{3}(k)+\frac{T_{s}}{L}\left(q_{3}(k)-q_{4}(k)-f(k)\right)
\end{gathered}
$$

With the elaboration of the inter-cell flow law can be defined the expressions for the inter cell flows $q_{1}, q_{2}$ and $q_{3}$ in the above equations (1.1, 1.2 and 1.3). Before the inter cell flows elaboration is given, a reasonable description of the congestion must be given further, since as we assumed above, the cells can be in either free 
or congested mode. Congestion is defined as the state of the traffic with high density rates, or with other words the density of that part of the highway expressed in cell is equal or higher than the critical density based on the fundamental diagram of relationship of flow and density. Referred to the mentioned diagram, can be noticed that the congested flow belongs to higher values of the density, above the critical density values where the flow drops down. That can be described with enormous number of vehicles travelling at low speeds and with short distance spaces between each other.

The common modes, used in analysis of researchers are the fully congested mode when the three cells are congested, denoted by $C C C$ mode, and free flow mode when the three cells are in free flow mode, denoted by FFF mode. The other middle modes that are out of the scope of this paper are those with last one and two cells in congested mode, written by $F C C$ and $F F C$, respectively. To emphasize the modes, the congested cells are highlighted further.

Now, for the $F F F$ mode, the densities of the cells are lower than the critical density and the inter cell flows are as follows:

$$
\begin{gathered}
q_{i}(k)=\min \left(v_{f i-1} \cdot \rho_{i-1}, Q_{i-1} w_{i}\left(\rho_{J}-\rho_{i}\right)\right) \text { or } \\
q_{2}(k)=\min \left(v_{f 1} \cdot \rho_{1}, Q_{1} w_{2}\left(\rho_{J}-\rho_{2}\right)\right)=v_{f 1} \cdot \rho_{1} \\
\mid q_{i+1}(k)=\min \left(v_{f i} \cdot \rho_{i}, Q_{i} w_{i+1}\left(\rho_{J}-\rho_{i+1}\right),\right) \\
q_{3}(k)=\min \left(v_{f 2} \cdot \rho_{2}, Q_{2} w_{3}\left(\rho_{J}-\rho_{3}\right)\right)=v_{f 2} \cdot \rho_{2}
\end{gathered}
$$

In $C C C$ mode, the densities of the cells are higher that the critical density, and the inter cell flows are:

$$
\begin{aligned}
& q_{i}(k)=\min \left(v_{f i-1} \cdot \rho_{i-1}, Q_{i-1}\right. \\
& \left.w_{i}\left(\rho_{J}-\rho_{i}\right)\right) \text { or } \\
& q_{2}(k)=\min \left(v_{f 1} \cdot \rho_{1}, Q_{1} w_{2}\left(\rho_{J}-\rho_{2}\right)\right)=w_{2}\left(\rho_{J}-\rho_{2}\right) \\
& q_{i+1}(k)=\min \left(v_{f i} \cdot \rho_{i}, Q_{i} w_{i+1}\left(\rho_{J}-\rho_{i+1}\right),\right) \\
& q_{3}(k)=\min \left(v_{f 2} \cdot \rho_{2}, Q_{2} w_{3}\left(\rho_{J}-\rho_{3}\right)\right)=w_{3}\left(\rho_{J}-\right. \\
& \left.\rho_{3}\right)
\end{aligned}
$$

After subtracting the expressions for inter cell flows (1.5 and 1.6) in the equations of densities (1.2, 1.3 and 1.4), for the $F F F$ mode, we have:

$$
\begin{aligned}
& \rho_{1}(k+1)=\rho_{1}(k)+\frac{T_{s}}{L}\left(q_{1}(k)-v_{f 1} \cdot \rho_{1}(k)+r(k)\right) \\
& \rho_{2}(k+1)=\rho_{2}(k)+\frac{T_{S}}{L}\left(v_{f 1} \cdot \rho_{1}(k)-v_{f 2} \cdot \rho_{2}(k)\right) \\
& \rho_{3}(k+1)=\rho_{3}(k)+\frac{T_{S}}{L}\left(v_{f 2} \cdot \rho_{2}(k)-q_{4}(k)-f(k)\right)
\end{aligned}
$$

And after subtracting the expressions for inter cell flows (1.7 and $1.8)$ in the equations of densities $(1.2,1.3$ and 1.4), for the $C C C$ mode, we have:

$$
\begin{aligned}
& \rho_{1}(k+1)=\rho_{1}(k)+\frac{T_{S}}{L}\left(q_{1}(k)-w_{2}\left(\rho_{J}-\rho_{2}(k)\right)+r(k)\right) \\
& \rho_{2}(k+1)=\rho_{2}(k)+\frac{T_{S}}{L}\left(w_{2}\left(\rho_{J}-\rho_{2}(k)\right)-w_{3}\left(\rho_{J}-\rho_{3}(k)\right)\right)
\end{aligned}
$$

$$
\rho_{3}(k+1)=\rho_{3}(k)+\frac{T_{s}}{L}\left(w_{3}\left(\rho_{J}-\rho_{3}(k)\right)-q_{4}(k)-f(k)\right)
$$

\section{State-Space presentation of CTM Model}

The state space presentation (particularly the state space, eq.1.17) of CTM based traffic densities of a highway segment in FFF mode differs from that of $C C C$ mode. [7].

What it characterizes the state space model of the traffic density based on CTM model, is implication of some other extension parts of the state space, $B_{q}$ which is the input matrix of upstream and downstream flows $q_{1}$ and $q_{4}$ respectively, $B_{r}$ the input matrix for on ramp and off ramp flows, $r$ and $f$ respectively that are applicable on the $F F F$ mode and input matrices, $B_{w}$ which takes into consideration the backward waves $w_{2}$ and $w_{3}$ and $B_{J}$ the input matrix of the jam density that are applicable on the state space model of the $C C C$ mode. (1.17) and (1.18)

$$
\begin{aligned}
& \rho_{(k+1) F F F}=A \rho_{(k)}+B_{u} q_{(k)}+B_{r, f}(r, f)_{(k)}+B w_{(k)} \\
& \rho_{(k+1) C C C}=A \rho_{(k)}+B_{u} u_{(k)}+B_{r, f}(r, f)_{(k)}+B_{J} \rho_{J(k)}+B w_{(k)}
\end{aligned}
$$

Where: $x(k+1)$ is the system state vector and in this paper, according to the CTM model, corresponds to the density in cell of cell $i$.

$A$ is the state matrix, $B$ is the input matrix, $u(k)$ is the input or control and $w_{k}$ represents the process noise.

They are assumed to be independent (of each other), white, and with normal probability distributions (Gaussian) as:

$p(w)^{\sim} N(0, Q)$ and $p(v)^{\sim} N(0, R)$.

From the system of equations in $(3.12,3.13$ and 3.14$)$ can be drawn (after some regulations finding partial derivatives of the functions of densities to the parameters previous densities $\rho(k)$, flows $q_{1}$ and $q_{4}$ and backward waves $w_{2}$ and $w_{3}$ which provide the elements of the respective matrices of the $i^{\text {th }}$ row that correspond to density of $i^{\text {th }}$ cell) the belonging matrices of the system matrices.

After bringing up together (eq. in 1.19 and 1.20 can be expanded to the form of state space: For $\underline{F F F}$ mode:

$$
\begin{aligned}
& \left(\begin{array}{l}
\rho_{1} \\
\rho_{2} \\
\rho_{3}
\end{array}\right)_{F F F}(k+1)=\left(\begin{array}{ccc}
1-v_{f 1} \cdot \frac{T_{s}}{l_{1}} & 0 & 0 \\
v_{f 1} \cdot \frac{T_{s}}{l_{2}} & 1-v_{f 2} \cdot \frac{T_{s}}{l_{2}} & 0 \\
0 & v_{f 2} \cdot \frac{T_{s}}{l_{3}} & 1
\end{array}\right) \cdot\left(\begin{array}{c}
\rho_{1} \\
\rho_{2} \\
\rho_{3}
\end{array}\right)(k)+ \\
& \left(\begin{array}{ccc}
\frac{T_{S}}{l_{1}} & 0 & 0 \\
0 & \frac{T_{s}}{l_{2}} & 0 \\
0 & 0 & \frac{T_{s}}{l_{3}}
\end{array}\right) \cdot\left(\begin{array}{c}
q_{1} \\
0 \\
-q_{4}
\end{array}\right)(k)+\left(\begin{array}{ccc}
\frac{T_{s}}{l_{1}} & 0 & 0 \\
0 & \frac{T_{s}}{l_{2}} & 0 \\
0 & 0 & \frac{T_{s}}{l_{3}}
\end{array}\right) \cdot\left(\begin{array}{l}
r \\
0 \\
0
\end{array}\right)(k)
\end{aligned}
$$

By recalling the standard state space models in (1.19) and (1.20), for completely free flow mode FFF and congested mode CCC respectively, there can be derived other variants, by changing the outflow from which is considered to have congestion the density formula, that is dictated by the backward speed and jam density of the downstream cell. 
For $\underline{C C C}$ mode:

$$
\begin{aligned}
& \left(\begin{array}{c}
\rho_{1} \\
\rho_{2} \\
\rho_{3}
\end{array}\right)_{C C C}(k+1)=\left(\begin{array}{cccc}
1 & w_{2} \cdot \frac{T_{s}}{l_{1}} & 0 & 0 \\
0 & 1-w_{2} \frac{T_{s}}{l_{2}} & w_{3} \cdot \frac{T_{s}}{l_{2}} \\
0 & 0 & 1-w_{3} \cdot \frac{T_{s}}{l_{3}}
\end{array}\right) . \\
& \left(\begin{array}{l}
\rho_{1} \\
\rho_{2} \\
\rho_{3}
\end{array}\right)(k)+\left(\begin{array}{ccc}
\frac{T_{s}}{l_{1}} & 0 & 0 \\
0 & \frac{T_{s}}{l_{2}} & 0 \\
0 & 0 & -\frac{T_{s}}{l_{3}}
\end{array}\right) \cdot\left(\begin{array}{c}
q_{1} \\
0 \\
q 4
\end{array}\right)(k)+\left(\begin{array}{ccc}
\frac{T_{s}}{l_{1}} & 0 & 0 \\
0 & \frac{T_{s}}{l_{2}} & 0 \\
0 & 0 & \frac{T_{s}}{l_{3}}
\end{array}\right) \cdot\left(\begin{array}{l}
r \\
0 \\
0
\end{array}\right)(k)+ \\
& \left(\begin{array}{ccc}
0 & -w_{2} \cdot \frac{T_{s}}{l_{1}} & 0 \\
0 & w_{2} \cdot \frac{T_{s}}{l_{2}} 0 & 0 \\
0 & 0 & w_{3} \cdot \frac{T_{s}}{l_{3}}
\end{array}\right) \cdot\left(\begin{array}{c}
\rho_{1 J} \\
\rho_{2 J} \\
\rho_{3 J}
\end{array}\right)(k)
\end{aligned}
$$

\section{A numerical example of the CTM model-Traffic density1.1. The building structure of the KF}

For the purpose of the demonstration of the CTM model, in this paper is performed a numerical example which is described below. For the sake of simplicity, are chosen the approximately the same freeway segment partitioning characteristics as that in earlier sections in order to do an interconnection with the laid state space model of traffic density. The system of performance measurements of the traffic road networks of the Californian state PeMs is used for traffic collection data and is considered a freeway link for on the street "Broadway Avenue", Stockton/San Francisco. The freeway is consisted from three cells with different lengths with one on-ramp on the first cell. (fig.1).

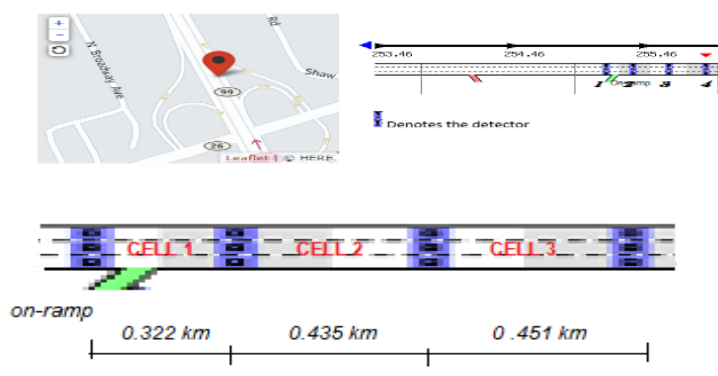

Fig. 1. Freeway segment with three cells

Calibrated parameters are given below [8].

\begin{tabular}{|c|c|c|c|c|c|c|}
\hline \multicolumn{6}{|c|}{ Table 1. Summary of traffic parameters for three cells } \\
\hline & $Q_{M}$ & $v f$ & $\rho c r$ & $\rho J$ & $w$ & $\begin{array}{c}\text { FF/ } \\
\text { /CC }\end{array}$ \\
\hline Cell 1 & 5580 & 84.8 & 65.8 & 248 & 30.6 & FF \\
\hline Cell 2 & 4176 & 96.8 & 43.1 & 248 & 20.3 & FF \\
\hline Cell 3 & 4268 & 106.7 & 40.0 & 248 & 20.5 & FF \\
\hline
\end{tabular}

The Kalman Filter consists of a set of mathematical
equations that provides an efficient recursive computation to estimate the state of a process by minimizing the mean of the squared error. [4] The KF estimates the value of the variable $\mathrm{x}$ at any time $(\mathrm{k}+1)$, represented by a linear stochastic equation.

$x_{k+1}=A x_{k}+B X_{k}+w_{k}$

Where: $A(k)$ is matrix which relates the state a time interval $k$ with the state at current time interval $k+1 . B(k)$ is matrix which relates the current state to the control input $X_{k}$.

The random variable $w$ represents noise in modelling process. It is assumed to be within normal probability distributions with zero mean and variance $\mathrm{Q}$ (Gaussian) as: $p(w)^{\sim} N(0, Q)$

The system measurement equation describes the relationship between system states and measurements. Acknowledging that measurements inevitably contain noise, the output equation is expressed as follows:

$\mathrm{Z}(\mathrm{k}+1)=\mathrm{H} \cdot \mathrm{x}(\mathrm{k})$,

$\tilde{Z}(k)$ is the measurement variable (outflow of vehicles from cell 3-measured by loop detector 2) $H(k)$ is the output matrix, and $v$ $(k)$ is the measurement noise variable. The errors in estimating $a$ priori and a posteriori states are defined as follows:

$P_{k}^{-}=x_{k}-\hat{x}_{k}^{-}$

$\mathrm{P}_{\mathrm{k}}=\mathrm{x}_{\mathrm{k}}-\hat{\mathrm{x}}_{\mathrm{k}}$

The $a$ priori and a posteriori estimate covariance is given by:

$P_{k}^{-}=E\left[e_{k}^{-}, e_{k}^{-T}\right]=A P_{k-1} \cdot A^{T}+Q$

$P_{k}=E\left[e_{k}, e_{k}^{T}\right)=\left(I-K_{k} H\right) \cdot P_{k}^{-}$

The KF estimates a posteriori state of the process using a linear combination of a priori state and a weighted difference between the actual measurement and the model measurement of the state. 
$\hat{x}_{k}=\hat{x}_{k}^{-}+K\left(z_{k}-H \cdot \hat{x}_{k}^{-}\right)$

$$
K_{k}=P_{k}^{-} H^{T}\left(H P_{k}^{-} H^{T}+R\right)^{-1}
$$

Based on the above equation, especially on (1.29), the $K F$ process can be divided in two steps ore phases. The first step is the prediction step and the second step is the correction step.

\section{Estimation with Kalman Filter}

In this section are described in detail the applied matrices to the algorithm of the $C T M-K F$ model. It is necessary to recall the equations state space of $C T M$ model (Section V.1) first and then to do an interconnection of it with the $K F$ algorithm equations. Since in our model, we are estimating the traffic densities of the three cells of the mentioned link, by the usage of the inputs values of the inflow $q_{1}$, output values $q_{4}$ and the flow from on ramp, then the state space vector of our algorithm are the densities $x=\left[x_{1}, x_{2}, x_{3}\right]=\left[\rho_{1}, \rho_{2}, \rho_{3}\right]^{T}$, the input vectors are

$$
\begin{gathered}
x_{u}=\left[q_{1}, 0, q_{4}\right]^{T} ; x_{r}=[q r, 0,0]^{T} \\
x_{k+1}=A x_{k}+B X_{U k}+w_{k}
\end{gathered}
$$

$$
\left(\begin{array}{l}
\rho_{1} \\
\rho_{2} \\
\rho_{3}
\end{array}\right)=A \cdot\left(\begin{array}{l}
\rho_{1} \\
\rho_{2} \\
\rho_{3}
\end{array}\right)+B u \cdot\left(\begin{array}{c}
q_{1} \\
0 \\
-q_{4}
\end{array}\right) B r \cdot\left(\begin{array}{l}
r \\
0 \\
0
\end{array}\right)
$$

On this paper we are going to use the measurement of the outflow from the cell three, that corresponds to the flow $q_{4}$ in the figure (3.1). Based on the fundamental diagram we model the traffic flow measurement through the densities on the last cell $\left(\rho_{3}\right)$ and the free flow speed on cell $3 v_{f 3}$ we will have $q_{\text {out }}=v_{f 3} \cdot \rho_{3}$ that is consistent with the equation (6.2)

$Z(k+1)=H \cdot x(k)$

$H=\left[\begin{array}{lll}0 & 0 & v_{f 3}\end{array}\right]$ and $x=\left(\begin{array}{c}\rho_{1} \\ \rho_{2} \\ \rho_{3}\end{array}\right), q_{\text {out }}-\left[\begin{array}{lll}0 & 0 & v_{f 3}\end{array}\right]\left(\begin{array}{c}\rho_{1} \\ \rho_{2} \\ \rho_{3}\end{array}\right)$

Where: $Q$-the model error covariance matrix which elements standard deviations of the density variables. The off diagonal elements are equal to zero while $R$ is the measurement or output error covariance. In this seminar paper, the matrices $Q$ and $R$ are assumed to be constant.[9]

$$
\mathrm{Q}=\left|\begin{array}{ccc}
\mathrm{w} & 0 & 0 \\
0 & \mathrm{w} & 0 \\
0 & 0 & \mathrm{w}
\end{array}\right| ; \mathrm{R}=\left|\begin{array}{ccc}
0 & 0 & 0 \\
0 & 0 & 0 \\
0 & 0 & \mathrm{v}
\end{array}\right|
$$

\section{Results and Conclusion}

Evaluation of the density values of cell is performed with discrete time intervals of $T_{s}=10$ seconds, where the initial values of the densities $\rho_{0}=\left[\rho_{10}, \rho_{20}, \rho_{30}\right]^{\mathrm{T}}$ and estimated covariance matrix $P_{o}$ are assumed. $T_{s}$ is chosen to be 10 second in order to fill the conditions $\mathrm{T}<L / v_{f}$, for proper work with system matrices, otherwise there will be obtained negative values of density parameters. For the purpose of the results evaluation, measured traffic densities for five minute intervals are used for comparison with the estimated densities with CTM model. The performance of the model was quantified by calculating the Mean Absolute Percentage Error (MAPE) given in (1.32).

$M A P E=\left[\frac{1}{n} \cdot \sum_{k=1}^{n} \mid 100 \frac{\rho_{\text {mod }}(k)-\rho_{\text {meas }}(k)}{\rho_{\text {meas }}(k)}\right] \cdot 100 \mid$

The MAPE results for CTM model for modes FFF -KF,

for Cell 1, Cell 2 and Cell3, $2 \%, 0.6 \%$ and $1 \%$ respectively, nd for CCC-KF, $14 \%$ on the three cells. The results of the estimated values by CTM model against the measured values of traffic density are also graphically presented on the below figure (3-5).

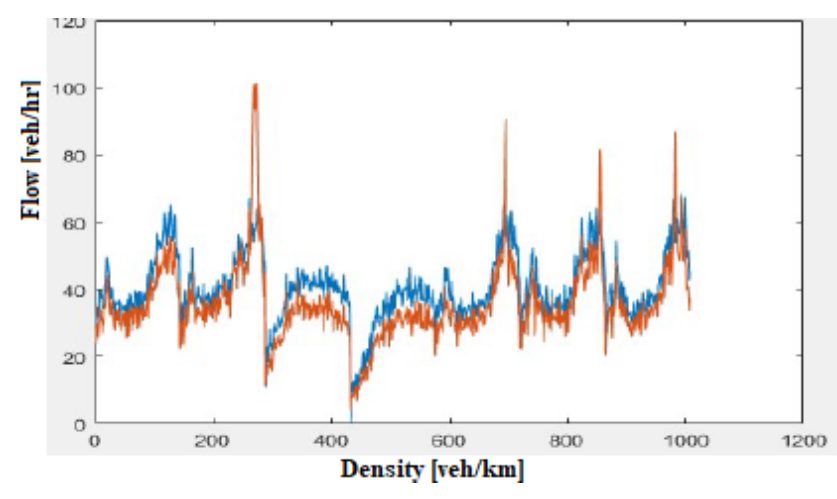

Fig.3. Graphic results densities of KF FFF and measurementsCell 1

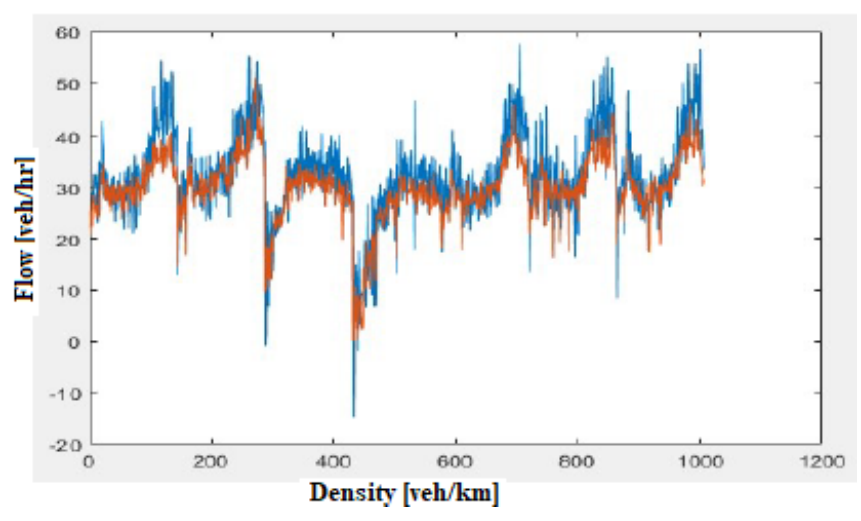

Fig.4. Graphic results densities of KF FFF and measurementsCell 2

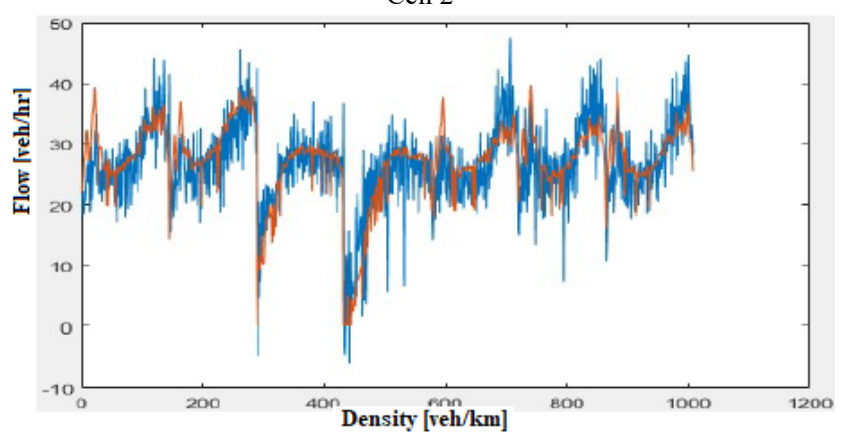

Fig.5. Graphic results densities of KF FFF and measurements- Cell3 


\section{References}

[1] Nicola George - Kathryn Kershaw, Road Use Statistics, Great Britain, 2016

[2] Ramp Metering: A Review of the Literature, E.D. Arnold ,Jr (1998)

[3] Chen X.M, Li, L; Stochastic Evolutions of Dynamic Traffic Flow Modeling and Applications, Chapter 2, http://www.springer.com/978-3-662-44571-6 Springer (2015)

[4] M.J .Lighthill ; G.B. Whitham "On Kinematic Waves, II: A theory of traffic flow on long crowded roads, Proceedings of the Royal Society of London, Series A-Mathematical and Physical Science.

[5] X. Sun, L. Munoz. R. Horowitz, A Mixture Kalman Filter Highway Congestion Mode and Vehicle Density Estimator and its Application (2004)

[6] Carlos F. Daganzo, "The Cell Transmission Model: Network Traffic", (1996)

[7] L. Munoz, X. Sun, R. Horowitz, L. Alvarez; "Traffic Density Estimation with the Cell Transmission Model1" (2003)

[8] G.B. Witham "Linear and Nonlinear Waves", Pure and Applied Mathematics, John Wiley Sons, New York City, USA, (1974)

[9] L. Munoz, X. Sun, R. Horowitz, D. Sun, G. Gomes; "Methodological calibration of the cell transmission model" Proceedings of the American Control Conference, Massachusetts 2004.

[10] A. Katsivalis, M. Papa Georgiou, 'The importance of Traffic Flow Modeling for Motorway Traffic Control', 2001 